\title{
THE RADIUS OF UNIVALENCE OF CERTAIN ANALYTIC FUNCTIONS
}

\section{S. D. BERNARDI}

1. Introduction. The writing of this paper has been motivated by a recent result of A. E. Livingston [5], and may also be regarded as a continuation of the investigation of certain classes of functions considered in [1] and [2].

Let $(S)$ denote the class of functions $f(z)=z+\sum_{2}^{\infty} a_{n} z^{n}$ which are regular and univalent in $E\{z:|z|<1\}$ and which map $E$ onto domains $D(f)$. We denote by $(C),\left(S^{*}\right)$, and $(K)$ the subclasses of $(S)$ where $D(f)$ are, respectively, close-to-convex, starlike with respect to the origin, and convex. It follows that $(K) \subset\left(S^{*}\right) \subset(C) \subset(S)$. Let $(P)$ denote the class of functions $p(z)$ which are regular and satisfy $p(0)=1, \operatorname{Re} p(z)>0$, for $z$ in $E$. In [2] the following theorem was proven.

TheOREM A. Let $f(z)=z+\sum_{2}^{\infty} a_{n} z^{n}$ be a member of $(C),\left(S^{*}\right)$, or $(K)$. Then

$$
F(z)=(c+1) z^{-c} \int_{0}^{z} t^{c-1} f(t) d t=z+\sum_{n=2}^{\infty}\left(\frac{c+1}{c+n}\right) a_{n} z^{n}
$$

is also a member of the same class for $c=1,2,3, \cdots$.

Theorem A represents a generalization of the corresponding theorem by R. J. Libera [4] for the special case $c=1$. Solving the relation (1.1) for the inverse function $f(z)$, we have

$$
f(z)=\left(\frac{1}{1+c}\right) z^{1-c}\left[z^{c} F(z)\right]^{\prime} .
$$

In [5] Livingston investigates the special case of (1.2) with $c=1$, $f(z)=\frac{1}{2}[z F(z)]^{\prime}$; if $F(z) \in\left(S^{*}\right)$ what is the radius $r_{0}$ of starlikeness of $f(z)$ ? Similar questions are answered under the assumption that $F(z)$ is in $(K)$ or in $(C)$. Livingston obtains the sharp result in each case to be $r_{0}=\frac{1}{2}$. Furthermore, he proved that if $F(z)=z+\cdots$ is regular and $\operatorname{Re}\left[F^{\prime}(z)\right]>0$ for $z$ in $E$, and $f(z)=\frac{1}{2}[z F(z)]^{\prime}$, then $\operatorname{Re}\left[f^{\prime}(z)\right]>0$ for $|z|<r_{1}=(\sqrt{ } 5-1) / 2$. This result is sharp.

It is the purpose of this paper to extend the results of Livingston to the general case $c=1,2,3, \cdots$ of the functions of (1.2). We shall

Received by the editors May 23, 1969. 
employ the same techniques used in [5] with necessary modifications needed to treat the more general case. All results obtained will be sharp.

Theorem 1. Let $F(z) \in\left(S^{*}\right), f(z)=[1 /(1+c)] z^{1-c}\left[z^{c} F(z)\right]^{\prime}, c=1,2$, $3, \cdots$. Then $f(z)$ is starlike for $|z|<r_{0}$ where

$$
r_{0}=\left[-2+\left(3+c^{2}\right)^{1 / 2}\right] /(c-1)
$$

for $c>1, r_{0}=1 / 2$ for $c=1$. This result is sharp.

Proof. Since $F(z) \in\left(S^{*}\right), \operatorname{Re}\left[z F^{\prime}(z) / F(z)\right]>0$ for $|z|<1$. Hence there exists a function $w(z)$, with $w(0)=0,|w(z)| \leqq|z|$, regular in $|z|<1$ such that

$$
\frac{z F^{\prime}(z)}{F(z)}=\frac{z^{c} f(z)-c \int_{0}^{z} t^{c-1} f(t) d t}{\int_{0}^{z} t^{c-1} f(t) d t}=\frac{1-w(z)}{1+w(z)}
$$

for $|z|<1$. Solving for $f(z)$,

$$
f(z)=\frac{(c+1)+(c-1) w(z)}{(1+w(z)) z^{c}}\left[\int_{0}^{z} t^{c-1} f(t) d t\right] .
$$

A computation yields

$$
\frac{z f^{\prime}(z)}{f(z)}=\frac{1-w(z)-z w^{\prime}(z)}{1+w(z)}+\frac{b z w w^{\prime}(z)}{1+b w(z)},
$$

where $b=(c-1) /(c+1)$. Equivalently,

$$
\frac{z f^{\prime}(z)}{f(z)}=\frac{1-w(z)}{1+w(z)}-(1-b)\left[\frac{z w^{\prime}(z)}{(1+w(z))(1+b w(z))}\right] .
$$

To show that $f(z)$ is starlike in $|z|<r_{0}$, we must show that $\operatorname{Re}\left[z f^{\prime}(z) / f(z)\right]>0$ in $|z|<r_{0}$. This condition is equivalent to

$$
(1-b) \operatorname{Re}\left[\frac{z w^{\prime}(z)}{(1+w(z))(1+b w(z))}\right]<\operatorname{Re}\left[\frac{1-w(z)}{1+w(z)}\right]
$$

for $|z|<r_{0}$. On the right-side of (1.3) substitute

$$
\operatorname{Re}\left[\frac{1-w(z)}{1+w(z)}\right]=\operatorname{Re}\left[\frac{(1-w(z))(1+w(z))}{|1+w(z)|^{2}}\right]=\frac{1-|w(z)|^{2}}{|1+w(z)|^{2}},
$$

and on the left side substitute 


$$
\begin{aligned}
\operatorname{Re}\left[\frac{z w^{\prime}(z)}{(1+w(z))(1+b w(z))}\right] & =\operatorname{Re}\left[\frac{z w^{\prime}(z)(1+w \bar{w}(z))(1+b \bar{w}(z))}{|(1+w(z))(1+b w(z))|^{2}}\right] \\
& \leqq \frac{\left|z w^{\prime}(z)(1+w(z))(1+b w(z))\right|}{|(1+w(z))(1+b w(z))|^{2}} \\
& =\frac{\left|z w^{\prime}(z)\right|}{|1+w(z)||1+b w(z)|} .
\end{aligned}
$$

Therefore the inequality (1.3) will be satisfied if

$$
\frac{(1-b)\left|z w^{\prime}(z)\right|}{|1+w(z)||1+b w(z)|}<\frac{1-|w(z)|^{2}}{|1+w(z)|^{2}}\left(|z|<r_{0}\right) .
$$

Applying the well-known result for bounded functions

$$
\left|w^{\prime}(z)\right| \leqq \frac{1-|w(z)|^{2}}{1-|z|^{2}} \quad(|z|<1)
$$

the inequality (1.4) will be satisfied if

$$
\frac{(1-b)|z|}{|1+w(z)||1+b w(z)|} \cdot \frac{1-|w(z)|^{2}}{1-|z|^{2}}<\frac{1-|w(z)|^{2}}{|1+w(z)|^{2}} .
$$

Simplifying, and writing $|z|=r$, we obtain

$$
\frac{(1-b) r}{1-r^{2}}<\left|\frac{1+b w(z)}{1+w(z)}\right| .
$$

Since $|w(z)| \leqq|z|=r<1,|(1+b w(z)) /(1+w(z))| \geqq(1+b r) /(1+r)$ so that (1.6) will be satisfied if

$$
\frac{(1-b) r}{1-r^{2}}<\frac{1+b r}{1+r}
$$

This reduces to

$$
1-2(1-b) r-b r^{2}>0, \quad \text { or }(c+1)-4 r-(c-1) r^{2}>0
$$

which gives the required root $r_{0}$ of Theorem 1 .

To see that the result is sharp for each $c$, consider the function $F(z)=z /(1-z)^{2}$. For this function we have

$$
\begin{aligned}
f(z) & =\left(\frac{1}{1+c}\right) \cdot\left(\frac{z[(1+c)-(c-1) z]}{(1-z)^{3}}\right), \\
\frac{z f^{\prime}(z)}{f(z)} & =\frac{(c+1)+4 z-(c-1) z^{2}}{(1-z)[(c+1)-(c-1) z]},
\end{aligned}
$$


so that $z f^{\prime}(z) / f(z)=0$ for $z=-r_{0}$. Thus $f(z)$ is not starlike in any circle $|z|<r$, if $r>r_{0}$. This completes the proof. Note that $\frac{1}{2} \leqq r_{0} \leqq 1$. The value $r_{0}=\frac{1}{2}$ is attained for $c=1$ when $f(z)=\frac{1}{2}[z F(z)]^{\prime}$. The value $r_{0}=1$ is attained for $c \rightarrow \infty$ when $f(z) \rightarrow F(z)$.

THEOREM 2. Let $F(z) \in(K), f(z)=[1 /(1+c)] z^{1-c}\left[z^{c} F(z)\right]^{\prime}, \quad c$ $=1,2,3, \cdots$. Then $f(z)$ is univalent for $|z|<1$ and is convex for $|z|<r_{0}$ where $r_{0}$ is the same as in Theorem 1. This result is sharp.

Proof. We have $(1+c) f^{\prime}(z)=(1+c) F^{\prime}(z)+z F^{\prime \prime}(z)$. Thus

$$
(1+c) \operatorname{Re} \frac{f^{\prime}(z)}{F^{\prime}(z)}=c+\operatorname{Re}\left[1+\frac{z F^{\prime \prime}(z)}{F^{\prime}(z)}\right]>c>0
$$

for $|z|<1$. Hence $f(z)$ is close-to-convex (relative to $F(z)$ ) and therefore is univalent in $|z|<1$. To show that $f(z)$ is convex for $|z|<r_{0}$, we note that

$$
z f^{\prime}(z)=\left(\frac{1}{1+c}\right) z^{1-c}\left[z^{c}\left(z F^{\prime}(z)\right)\right]^{\prime} .
$$

Since $F(z) \in(K), z F^{\prime}(z) \in\left(S^{*}\right)$. Therefore, by Theorem $1, z f^{\prime}(z)$ is starlike for $|z|<r_{0}$ and hence $f(z)$ is convex for $|z|<r_{0}$. The result is sharp, for each $c$, for the function $F(z)=z /(1-z)$. For this function we have

$$
\begin{aligned}
& f(z)=\frac{(1+c) z-c z^{2}}{(1+c)(1-z)^{2}} \\
& J(z)=1+\frac{z f^{\prime \prime}(z)}{f^{\prime}(z)}=\frac{(1+c)+4 z-(c-1) z^{2}}{(1-z)[(1+c)-(c-1) z]},
\end{aligned}
$$

so that $J(z)=0$ for $z=-r_{0}$. Thus $f(z)$ is not convex in any circle $|z|<r$, if $r>r_{0}$.

THEOREM 3. Let $F(z)$ be close-to-convex with respect to $G(z), c$ $=1,2,3, \cdots$.

$$
f(z)=\left(\frac{1}{1+c}\right) z^{1-c}\left[z^{c} F(z)\right]^{\prime} ; \quad g(z)=\left(\frac{1}{1+c}\right) z^{1-c}\left[z^{c} G(z)\right]^{\prime} .
$$

Then $f(z)$ is close-to-convex with respect to $g(z)$ for $|z|<r_{0}$ where $r_{0}$ is the same as in Theorem 1. This result is sharp.

Proof. By definition of close-to-convexity [3], there exists a real $\beta,|\beta|<\pi / 2$, such that $\operatorname{Re}\left[e^{i \beta} z F^{\prime}(z) / G(z)\right]>0$ for $|z|<1$, where $G(z) \in\left(S^{*}\right)$. By Theorem $1, g(z)$ is starlike for $|z|<r_{0}$. It is sufficient 
then to prove that $\operatorname{Re}\left[e^{i \beta} z f^{\prime}(z) / g(z)\right]>0$ for $|z|<r_{0}$. We have

$$
e^{i \beta}\left[\frac{z F^{\prime}(z)}{G(z)}\right]=p(z) \cos \beta+i \sin \beta
$$

where $p(z) \in(P)$. From this we obtain

$$
e^{i \beta}\left[z^{1+c} F^{\prime}(z)\right]^{\prime}=\cos \beta\left[p(z)\left(z^{c} G(z)\right)^{\prime}+p^{\prime}(z)\left(z^{c} G(z)\right)\right]+i \sin \beta\left(z^{c} G(z)\right)^{\prime} .
$$

A computation then gives

$$
\begin{aligned}
e^{i \beta}\left[\frac{z f^{\prime}(z)}{g(z)}\right] & =e^{i \beta} \frac{\left[z^{1+c} F^{\prime}(z)\right]^{\prime}}{\left[z^{c} G(z)\right]^{\prime}} \\
& =\cos \beta\left[p(z)+p^{\prime}(z) \frac{z^{c} G(z)}{\left(z^{c} G(z)\right)^{\prime}}\right]+i \sin \beta .
\end{aligned}
$$

Taking real parts we have

(1.10) $\operatorname{Re}\left[e^{i \beta} \frac{z f^{\prime}(z)}{g(z)}\right] \geqq \cos \beta\left[\operatorname{Re} p(z)-\left|p^{\prime}(z)\right|\left|\frac{z^{c} G(z)}{\left(z^{c} G(z)\right)^{\prime}}\right|\right]$.

If in (1.5) we substitute $w(z)=(p(z)-1) /(p(z)+1)$ we obtain the known result

$$
\left|p^{\prime}(z)\right| \leqq \frac{2 \operatorname{Re} p(z)}{1-|z|^{2}} \quad(|z|<1) .
$$

Applying (1.11), the inequality (1.10) gives

(1.12) $\quad \operatorname{Re} e^{i \beta} \frac{z f^{\prime}(z)}{g(z)} \geqq \operatorname{Re} p(z)\left[1-\frac{2}{1-|z|^{2}}\left|\frac{z^{c} G(z)}{\left(z^{c} G(z)\right)^{\prime}}\right|\right] \cos \beta$ for $|z|<1$. We note that

$$
M(z)=\frac{z\left(z^{c} G(z)\right)^{\prime}}{\left(z^{c} G(z)\right)}=c+\frac{z G^{\prime}(z)}{G(z)},
$$

from which we obtain

$$
|M(z)| \geqq \operatorname{Re} M(z) \geqq c+\frac{1-|z|}{1+|z|}=\frac{(c+1)+(c-1)|z|}{1+|z|}
$$

for $|z|<1$. We then have

$$
\left|\frac{z}{M(z)}\right|=\left|\frac{z^{c} G(z)}{\left(z^{c} G(z)\right)^{\prime}}\right| \leqq \frac{|z|+|z|^{2}}{(c+1)+(c-1)|z|} .
$$


Applying (1.13), the inequality (1.12) gives

$$
\operatorname{Re} e^{i \beta} \frac{z f^{\prime}(z)}{g(z)} \geqq \operatorname{Re} p(z)\left[1-\left(\frac{2}{1-|z|^{2}}\right) \frac{|z|+|z|^{2}}{(c+1)+(c-1)|z|}\right] \cos \beta
$$

for $|z|<1$. Writing $|z|=r$ and simplifying,

$$
\operatorname{Re} e^{i \beta} \frac{z f^{\prime}(z)}{g(z)} \geqq \operatorname{Re} p(z)\left[\frac{(c+1)-4 r-(c-1) r^{2}}{(1-r)[(c+1)+(c-1) r]}\right] \cos \beta .
$$

The right side of (1.14) is positive provided $(c+1)-4 r-(c-1) r^{2}>0$ or $r<r_{0}$. The result is sharp, for each $c$, for the function $F(z)=G(z)$ $=z /(1-z)^{2}$ which belongs to the class $\left(S^{*}\right)$ and therefore to the class $(C)$. Moreover $z f^{\prime}(z) / g(z)=$ same expression as the right side of (1.7), with the same result at $z=-r_{0}$.

Note that Theorem 3 may be restated without any reference to the functions $g(z)$ and $G(z)$. Let $F(z) \in(C), f(z)=[1 /(1+c)] z^{1-c}\left(z^{c} F(z)\right)^{\prime}$, $c \geqq 1$. Then $f(z)$ is close-to-convex for $|z|<r_{0}$.

Theorem 4. Let $F(z)=z+\cdots$ be regular and have the property $\operatorname{Re} F^{\prime}(z)>0$ for $|z|<1, f(z)=[1 /(1+c)] z^{1-c}\left[z^{c} F(z)\right]^{\prime}, c=1,2,3, \cdots$. Then $\operatorname{Re} f^{\prime}(z)>0$ for $|z|<r_{1}=\left[-1+\left(2+2 c+c^{2}\right)^{1 / 2}\right] /(1+c)$. This result is sharp.

Proof. Let $F^{\prime}(z)=p(z)$, where $p(0)=1$ and $\operatorname{Re} p(z)>0$ for $|z|<1$. We then have

$$
(1+c) f^{\prime}(z)=z F^{\prime \prime}(z)+(1+c) F^{\prime}(z)=(1+c) p(z)+z p^{\prime}(z) .
$$

Applying the estimate (1.11) and writing $|z|=r$ we obtain

$$
\begin{aligned}
(1+c) \operatorname{Re} f^{\prime}(z) & \geqq(1+c) \operatorname{Re} p(z)-r\left|p^{\prime}(z)\right| \\
& \geqq \operatorname{Re} p(z)\left[(1+c)-\frac{2 r}{1-r^{2}}\right] \\
& =\operatorname{Re} p(z)\left[\frac{(1+c)-2 r-(1+c) r^{2}}{1-r^{2}}\right] .
\end{aligned}
$$

The right side of $(1.15)$ is positive provided $(1+c)-2 r-(1+c) r^{2}$ $>0$, or $r<r_{1}$. The result is sharp, for each $c$, for the function $F(z)$ $=-z-2 \log (1-z)$. For this function we have

$$
\operatorname{Re} F^{\prime}(z)=\operatorname{Re}[(1+z) /(1-z)]>0
$$

for $|z|<1$, 


$$
\begin{aligned}
& f(z)=\frac{1}{1+c}\left[\frac{(c+1) z^{2}-(c-1) z}{1-z}-2 c \log (1-z)\right], \\
& f^{\prime}(z)=\frac{1}{1+c}\left[\frac{(1+c)+2 z-(1+c) z^{2}}{(1-z)^{2}}\right] .
\end{aligned}
$$

We then have $f^{\prime}(z)=0$ when $(1+c)+2 z-(1+c) z^{2}=0$, or $z=-r_{1}$. Thus $\operatorname{Ref}^{\prime}(z) \ngtr 0$ in any circle $|z|<r$ if $r>r_{1}$.

Note that $r_{1} \geqq r_{0}$. This is to be expected, since the class of functions $F(z)$ such that $F^{\prime}(z) \in(P)$ is a subclass of the class $(C)$. We also note that in the case where $F(z) \in(K)$, the function $f(z)$ $=[1 /(1+c)] z^{1-c}\left[z^{c} F(z)\right]^{\prime}=[1 /(1+c)]\left[c F(z)+z F^{\prime}(z)\right], \quad c \geqq 1$, has a simple geometrical interpretation in terms of subordination. Let $c_{r}$ be the convex image curve of $|z|=r<1$ by the function $F(z) \in(K)$. Since $z F^{\prime}(z)$ is an outward normal vector to $c_{r}$ at the point $F(z)$, we have $F(z) \prec a F(z)+b e^{i \alpha} z F^{\prime}(z)$ for $a \geqq 1, b \geqq 0,|\alpha| \leqq \pi / 2$. As a special case we obtain $F(z) \prec[(1+c) / c] f(z)$.

\section{REFERENCES}

1. S. D. Bernardi, Special classes of subordinate functions, Duke Math. J. 33 (1966), 55-67. MR 32\#5858.

2. - Convex and starlike univalent functions, Trans. Amer. Math. Soc. 135 (1969), 429-446. MR 38\#1243.

3. W. Kaplan, Close-to-convex schlicht functions, Michigan Math. J. 1 (1952), 169185. MR 14, 966.

4. R. J. Libera, Some classes of regular univalent functions, Proc. Amer. Math. Soc. 16 (1965), 755-758. MR 31\#2389.

5. A. E. Livingston, On the radius of univalence of certain analytic functions, Proc. Amer. Math. Soc. 17 (1966), 352-357. MR 32\#5861.

NEW YORK UNIVERSITY 